\section{Pharmacology of epidural anaesthesia}

Sheldon H. Roth PHD, Departments of Pharmacology \& Therapeutics and Anaesthesia, Health Sciences Centre, University of Calgary, Calgary, Alberta, Canada, T2N 4N1.

The epidural space extends from the base of the skull to the coccyx. It contains semi-fluid lobulated fat (areolar tissue) which is highly vascular, a plexus of venous blood vessels, lymphatics and fibrous connections between the dura and spinal column. ${ }^{1}$ Fibrous tissue is more extensive in the elderly. The volume of the epidural space varies with the region (i.e., shape) and contents of the vertebral canal; the areas of largest volume are the caudal canal and lumbar region. Capacity also varies with changes in position or posture and with volume occupied by venous blood vessels. The dura was originally thought to be impermeable to most substances and to act as a barrier to the central nervous system; this has been found to be untrue. ${ }^{2}$ The dura is very permeable in certain areas to anaesthetics and narcotics: toxic symptoms may be observed at high doses. The limit of the vertebral epidural space at the base of the skull is anatomical rather than functional.

A number of sites have been proposed to explain the action of epidural anaesthetics, i.e., mixed spinal nerves in paravertebral spaces, dorsal root ganglia, spinal roots within dural sleeves, and the spinal cord. The most important site appears to be the spinal cord and nerves in the subarachnoid space. Anaesthetic enters this region by diffusing across the dura or possibly by retrograde diffusion through intervertebral foramina. It is also likely that the nerve roots and dorsal root ganglia in the paravertebral area are affected as a result of diffusion of anaesthetic outwardly through intervertebral foramina, thus producing multiple nerve blocks. In caudal anaesthesia, the major action is believed to be a blockade of nerves as they pass through the epidural space, but diffusion via sacral foramina is also involved. There are many similarities between spinal anaesthesia and lumbar epidural anaesthesia which suggest that the subarachnoid space is involved in the mechanism of both. However, there is one major difference. Epidural anaesthesia does not exhibit a zone of differential sympathetic blockade compared to sensory blockade; but a zone differential occurs between sensory and somatic motor blockade (approximately 4-5 spinal segments).

Local anaesthetics reversibly prevent generation and conduction of electrical impulses in neurons (and other excitable membranes) by decreasing the large transient increase in permeability to sodium ions. ${ }^{3}$ The minimum effective concentration $(\mathrm{Cm})$ for local anaesthetics can vary from neuron to neuron. This differential sensitivity generally is dependent on size of the fibre; small fibres are more sensitive than large and require a longer period for recovery. Sensory pain fibres are usually the first to be blocked, followed by fibres that transmit sensations of cold, warmth, touch and deep pressure. High concentrations will block sympathetic, somatic sensory and somatic motor fibers. Changes in neural and perineural tissue are believed to be the cause of observed differences in susceptibility to epidural anaesthesia with age. Decreases in neural population, conduction velocity and inter-Schwann cell gap width, as well as changes in connective tissue mucopolysaccharides, result in lower $\mathrm{Cm}$ values.

Distribution in the epidural space of injected anaesthetic solution determines the spread, i.e., segmental level, of anaesthesia. This is primarily dependent on the volume of drug injected and the capacity of the epidural space. Position of the patient and gravity do not significantly influence distribution. Concentration of local anaesthetic determines the degree of block and type and number of fibres affected. The mechanism of action of epidural opiate or narcotic anaesthetics/analgesics is postulated to involve the specific interaction of these agents with opiate (nociceptive) receptors in the spinal cord, thereby modifying the response to painful stimuli. ${ }^{4,5}$

The combination of large volumes and high concentrations of anaesthetics into the highly vascularized epidural region can result in significant plasma concentrations of local anaesthetics. ${ }^{6}$ This is of great importance during labour and delivery. ${ }^{7}$ All local anaesthetics cross the placenta and therefore enter the foetal circulation, and may cause 
neurobiological effects in the neonate. ${ }^{8}$ At term the vascularity of the epidural space is increased, thus enhancing the possibility of systemic drug absorption. Epinephrine $(1: 200,000)$ is commonly employed to decrease systemic absorption. However, it should be noted that resultant plasma concentrations of epinephrine may be sufficient to produce peripheral vasodilation through beta-adrenergic stimulation.

\section{References}

1 Bromage PR. Epidural Analgesia, Philadelphia, W.B. Saunders Co, 1978.

2 Moore RA, Bullingham RES, MCQuay HJ et al. Dural permeability to narcotics: in vitro determination and application to extradural administration. Br J Anaesth. 1982; 54: 1117-28.

3 Gilman AG, Goodman LS, Gilman A. The Pharmacological Basis of Therapeutics, 6th ed, New York, MacMillan Publishing Co. Inc., 1980.

4 Justins DM, Knott C, Luthman J, Reynolds F. Epidural versus intramuscular fentanyl. Analgesia and pharmacokinetics in labour. Anaesthesia 1983; 38: 937-42.

5 Welchew EA, Thornton JA. Continuous thoracic epidural fentanyl. A comparison of epidural fentanyl with intramuscular papaveretum for postoperative pain. Anaesthesia 1982; 37: 309-16.

6 Takasaki $M$. Blood concentrations of lidocaine, mepivacaine and bupivacaine during caudal analgesia in children. Acta Anaesthesiol Scand 1984; 28: 211-4.

7 Abboud TK, Afrasiabi A, Sarkis $F$ et al. Continuous infusion epidural analgesia in parturients rcceiving bupivacaine, chloroprocaine or lidocaine - maternal, fetal, and neonatal effects. Anesth Analg 1984; 63: 421-8.

8 Kuhnert BR, Harrison MJ, Linn PL, Kuhnert PM. Effects of maternal epidural anaesthesia on neonatal behavior. Anesth Analg 1984; 63: 301-8

\section{What is the place of bupivacaine in obstetric epidural analgesia?}

George A. Albright MD, Department of Anaesthesia, Stanford University School of Medicine, Stanford, California 94305.

Bupivacaine is the most widely used local anaesthetic in obstetric anaesthesia because of its properties of long duration of action, weak motor block in analgesic concentrations, and greater safety for mother and foetus. Bupivacaine was reputed to be safer for the foetus and neonate because: the low umbilical blood/maternal vein ratio suggested reduced placental transfer of drug; the drug was reported to have a short half-life in the neonate (60-90 $\mathrm{min}$ ); and there was an absence of neurobehavioural effects. However, none of these advantages has stood the test of time. ${ }^{1-3}$ Additionally, unpublished case histories suggest that bupivacaine induced seizures without depression of the maternal cardiovascular system may result in foetal asphyxia and poor outcome, presumably from uterine hyperactivity and/or uterine artery spasm.

Bupivacaine may occasionaly have a slow, unpredictable spread of analgesic effect $(30-40 \mathrm{~min})$, result in spotty analgesia, regress in $60-90 \mathrm{~min}$, or progress to higher spinal segments after an established block. Use of epinephrine in obstetrics is controversial, despite the fact that there are no clinical studies demonstrating any adverse neonatal effects. ${ }^{4,5}$ In the author's experience, the efficacy of bupivacaine can be improved by the use of a 50/50 mixture of 0.5 per cent bupivacaine and one per cent lidocaine or mepivacaine with epinephrine; a 10 $12 \mathrm{ml}$ dose of this mixture provides fast, reliable onset and longer duration of labour pain relief and facilitates perineal anaesthesia.

Bupivacaine 0.75 per cent, in a dose of 18 $20 \mathrm{ml}$, was often used for Caesarean section but reports of cardiac arrest with this concentration and dose resulted in a warning against its use in 